\title{
Models and Testing - A Recipe for Improved Effectiveness?
}

\author{
Paul Baker \\ Motorola Ltd. \\ Motorola's Corporate Software Excellence Team \\ Basingstoke, Hampshire, RG22 4PD, United Kingdom \\ Paul.Baker@motorola.com
}

\begin{abstract}
In an ongoing effort to reduce development costs in spite of increasing system complexity, Motorola has been a long-time adopter of Model-Driven Engineering practices. The foundation of this approach is the creation of rigorous models throughout the development process, thereby, enabling the introduction of automation into the development life cycle and supporting frameworks.
\end{abstract}

\section{Introduction}

In this talk we present Motorola's experiences over the past 15 years in using models [1][2] to improve testing effectiveness from defect prevention through to test configuration. We present the motivation(s) for using models and in particular their impact on product testing and quality. Specifically, we present and discuss cases and metrics where models have been used for:

- Defect Prevention. We present the approaches that have been employed for the early discovery of defects through the application of requirements verification techniques and the lessons learnt from doing so. In addition, we introduce recent work on trying to fix identified defects automatically through the application of inference [3][4][5];

- Early testing of designs through the use of co-simulation, as well as, experiences from previous usage of formal verification techniques[6];

- Aiding test development and reuse. In this case we discuss some of the issues encountered during general test development and elaborate why modeling concepts are useful in overcoming some of these obstacles [7];

- Enabling test generation and automation. It is well understood that models can lend themselves to test generation. We present some of the issues learnt and also their application within the context of existing modeling and test standards, such as UML, TTCN-3, MSC etc.;

- Model-driven testability. Here we introduce recent work on using models for the systematic understanding of testability concerns [8], which are crucial for cost effective test automation.

We also present lessons learnt from deploying model-related technologies and the different business strategies that have been used to effect their deployment, e.g. maturity 
models, aspects, and services. In doing so, we discuss the lessons learnt and directions/challenges moving forward with the perspective of a large testing technology portfolio.

\section{References}

[1] Baker, P., Loh, S., Weil, F.: Model-Driven Engineering in a Large Industrial Context Motorola Case Study. In: Briand, L.C., Williams, C. (eds.) MoDELS 2005. LNCS, vol. 3713, pp. 476-491. Springer, Heidelberg (2005)

[2] Weigert, T., Weil, F., Marth, K., Baker, P., Jervis, C., Dietz, P., Gui, Y., Van de Berg, A., Fleer, K., Nelson, D., Wells, M., Mastenbrook, B.: Experiences in Deploying ModelDriven Engineering. In: Gaudin, E., Najm, E., Reed, R. (eds.) SDL 2007. LNCS, vol. 4745, Springer, Heidelberg (2007)

[3] Baker, P., Bristow, P., Burton, S., King, D., Jervis, C., Mitchell, B., Thomson, R.: Detecting and Resolving Semantic Pathologies in UML Sequence Diagrams. In: Joint 10th European Software Engineering Conference and 13th ACM SIGSOFT Symposium on the Foundations of Software Engineering, Lisbon, September 2005, pp. 50-59 (2005)

[4] Mitchell, B.: Resolving Race Conditions in Asynchronous Partial Order Scenarios. IEEE Transactions on Software Engineering, TSE-0039-0205 31(9), 767-784 (2005)

[5] Baranov, S., Jervis, C., Kotlyarov, V., Letichevsky, A., Weigert, T.: UML for Real Design of Embedded Real-Time Systems, pp. 323-342 ISBN 978-1-4020-7501-8

[6] Baker, P., Jervis, C.: Early UML Model Testing using TTCN-3 and the UML Testing Profile. In: TAICPART: Testing, Academic, and Industrial Conference, Practice and Research, IEEE Computer Society Press, Los Alamitos ISBN 0-7695-2984-4

[7] Baker, P., Dai, Z.R., Grabowski, J., Haugen, O., Schieferdecker, I., Williams, C.E.: Model-Driven Testing Using the UML Testing Profile. Springer, Heidelberg (2007)

[8] Baker, P., Jervis, C.: Early Model Testing and Testability Analysis using UML. Software Testing, Verification and Reliability (STVR) (submitted) 